\title{
Monotonic Vector Space Model(II): Application in System Capability Indicator Analysis and Design
}

\author{
Jianwen Hu \\ National University of Defense Technology, Changsha, China \\ Email: hjwc3i@sina.com
}

Received December 7, 2013; revised January 7, 2014; accepted January 14, 2014

Copyright (c) 2014 Jianwen Hu. This is an open access article distributed under the Creative Commons Attribution License, which permits unrestricted use, distribution, and reproduction in any medium, provided the original work is properly cited. In accordance of the Creative Commons Attribution License all Copyrights (C) 2014 are reserved for SCIRP and the owner of the intellectual property Jianwen Hu. All Copyright (C) 2014 are guarded by law and by SCIRP as a guardian.

\begin{abstract}
The application of some MVS operations (each dimension of which corresponds to a capability indicator) in system capability analysis and design, including capability indicator requirement analysis, effectiveness analysis, sensibility analysis, fuzzy analysis, stability analysis, capability optimization design, etc., is discussed in the second paper of this series of papers. And some MVS-based models and algorithms for capability analysis and design are put forward. Finally, an example of capability analysis and optimization design is given for explaining the usages of related models and algorithms.
\end{abstract}

\section{KEYWORDS}

Monotonic Vector Space; System Capability Indicators; Analysis; Optimization Design

\section{Introduction}

System capability indicator analysis and design are very important and also difficult for system development and system engineering. Many practical methods have been proposed and will be introduced in the following.

\subsection{The Representative System Capability Indicator Analysis Methods}

- Index method. It is a traditional method for weapon system capability indicator analysis. This method is suitable for synthesis and easy to understood. However, the index formula and weight influenced by analyzer are difficult to be determined. And the analysis result of index method is to some extent ambiguous.

- ADC model [1]. Based on the principle of division and transformation of system state, this method proposed by WEISIC is able to give clear quantitative analysis result. However, the calculation overload will exponentially grow with the increasing of system dimension number. Furthermore, other models and algorithms are required to get the capability vector $\mathrm{C}$ of ADC model.

- Weight-based multi-attributes analysis method [2,3]. This type of method such as AHP [4], ELECTRE, LINAMP, etc., determines weight of each indicator firstly. Then, all indicators are synthesized according to each of their weights in a linear way. Obviously, they are regardless of the mutual influence among indicators and so fail to reveal the nonlinearity of complex system. And furthermore their analysis results are also unclear.

- SEA method [5]. This method compares system capabilities with mission requirements in a common attribute space and emphasizes the whole characteristic of complex system. This comparison leads to the evaluation of partial measures of effectiveness that are then combined to yield a global measure. But system mission requirement loci used by this method are difficult to acquire.

\subsection{System Capability Indicator Design Method}

- Linera programming methods. This kind of method is traditional and neat. Many complex problems (espe- 
cialy with nolineartity and uncertainty) have to be over trivialized so as to be solved by these neat methods. Therefore, these methods are not directly effective for many complex problems.

- Capability-based Planning (CPB) [6]. CPB method emphasizes the flexibility, adaptiveness and robustness of capability and usually adopts exploratory analysis strategy. Compared with programming method, $\mathrm{CPB}$, as a flexible and non-neat method, is more suitable for complex problems.

Monotonic vector space (MVS) model is used for system capability indicator analysis and design in this paper. Since each dimension of MVS corresponds to one capability indicator, the MVS, therefore, is also called Monotonic Indicator Space (MIS) here. And likewise there exist some monotonic mappings between capability indicators and requirement measures in MIS.

\subsection{Assumptions Adopted in This Paper}

A monotonic relationship between the system capability indicators and the system requirement measures is assumed in this paper. It must be noted that system capability indicators are different from system parameters. For example, the velocity of a certain aircraft is not a capability indicator, but the maximal (or minimal) velocity of the aircraft is a system capability indicator. This assumption is usually in accordance with the practical situation in many cases. For example, in reference [5], there exists a monotonic relationship between the capability indicators (including reliability indicator $R$, survivability indicator $S$, delay time indicator $T$, and link capacity indicator $K$ ) and the requirement measure, i.e. the ratio of kill to loss. In reference [7], there also exists a monotonic relationship between the capability indicators (including link reliability indicator and delay time indicator) and the requirement measure (the kill probability). Actually, similar monotonic relationship between the capability indicators and the requirement measure can be found in many practical cases introduced by references [8-12].

In addition, the weak capability is assumed to be a subset of the stronger one. Thus, if a system with weak capability meets some certain mission requirements, the one with stronger capability will be certain to meet these mission requirements. For example, if an aircraft with the maximal cruise velocity of $1800 \mathrm{~km} / \mathrm{h}$ can arrive at a certain location in one hour (a requirement measure), the one with maximal cruise velocity of 3000 $\mathrm{km} / \mathrm{h}$ will definitely be able to arrive at the same location in one hour as long as the other capability indicators of these two aircrafts are equal.

The contents of system capability indicator analysis and design based on MIS include indicator requirement analysis, effectiveness analysis, sensitivity analysis, fuzzy analysis, relativity analysis, finance-based optimization design and time-based optimization design. The mutual relationships among these contents are shown in Figure 1. Obviously, system indicator analysis is the start point of system capability indicator analysis and design. And in the following sections, the related models and algorithms for system capability indicator analysis and design will be dealt with in detail.

\section{MIS-Based System Capability Indicator Analysis}

\subsection{System Capability Indicator Requirement Analysis}

The partitioning operation in MVS introduced by the first part of this series paper is used to produce the system capability requirement locus. For example, the dimensions of a monotonic indicator space $P$ for a ground-to-air missile system may include the searching radar detecting capability indicator dimension $K_{1}$, delay time indicator dimension $T$, and tracking radar detecting capability indicator dimension $K_{2}$. So, the equation

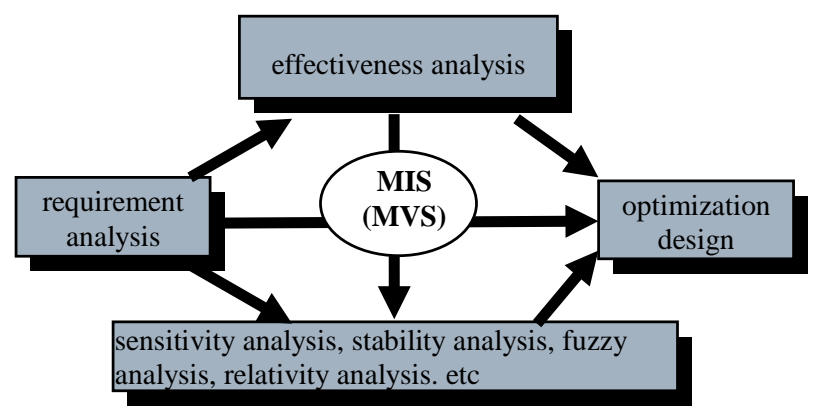

Figure 1. MVS-based system capability indicators analysis and design. 
$u=f\left(K_{1}, T, K_{2}\right)$ describes the relationship between these three system capability indicators (i.e., $K_{1}$, $T$, and $K_{2}$ ) and one requirement measure $u$ (the kill probability). The function $f$ may adopt several different forms, each of which corresponds to a different context. For example, $f_{1}$ and $f_{2}$ correspond to the different contexts in which the enemy stealth aircraft is respectively type A and type B. Function $f$ is obviously monotonic, that is to say, the value of $u$ (the kill probability) will monotonically increase or decrease with one of three system capability indicators changing monotonically while the others maintaining to be constants. Because the monotonic relationship between capability indicators ( $K_{1}$ and $K_{2}$ ) and the requirement measure (the kill probability) is different from that between capability indicator $(T)$ and the kill probability, so, $K_{1}$ and $K_{2}$ are called monotonic increasing indicators and $T$ called monotonic decreasing indicator.

Every dimension in MIS can be continuous or discrete type. System capability indicators are usually of continuous type as all the above mentioned ones. For discrete-type indicators, if its range is large enough, it still can be considered as continuous type just like many integer programming problems can be solved by general linear programming model. In this paper, all the dimensions of MIS are assumed to be continuous. The purpose of system capability indicator requirement analysis is to find out the MVRL or MIRL (monotonic indicator requirement locus) which can then be acquired with the algorithm proposed by the first part of this series papers. Figure 2 shows the MIRL for the above-mentioned ground-to-air missile system. There are 3 MIS dimensions, each of which corresponds to a capability indicator. The requirement measure $u$ (the kill probability for enemy invading stealth aircraft of type A and type B) is assumed as $u \geq 0.7$. The simulation calculation realizes the mapping from capability indicators to requirement measure. The MIRLs are produced by the partitioning algorithm at first and then synthesizing operation is used to produce the intersection of these multiple MIRLs which correspond to multiple requirements. According to the algorithm for producing MIRL, the algorithm output is a heap tree consisting of MIHs and MEHs. The MIHs compose the MIRL.

\subsection{Effectiveness Analysis for the Indicators of Stochastic Type}

Due to the uncertainty of the system capability indicators, it is usually difficult to represent them precisely. So the probability distribution function is applied to represent the system capability indicators. We represent a system capability indicator with such equation as $p_{i}=p_{i e}+p_{i s}$, where $p_{i e}$ and $p_{i s}$ respectively denote the expected value and a stochastic variable. Furthermore, a union probability density function $f(p)$ where $p$ is the vector in the MIS is used to describe the system capability indicators. The system effectiveness is denoted as $E$ and its expression is $E=\int_{P l} f(p) \mathrm{d} p$, where $P l$ is the MIRL. It can be easily known that the range of $E$ is $[0,1]$. As the system effectiveness, $E$ reflects the extent to which system meets the requirements. If the MIRL is approximated by the hyperboxes, i.e. $P l \approx \bigcup_{j=1}^{N} H_{j}$, where $H_{j}$ is the jth hyperbox and $N$ is the number of hyperboxes, the expression of $E$ will change into $E \approx \sum_{j=1}^{N} \int_{H_{j}} f(p) \mathrm{d} p$.

\subsection{Sensitivity and Relativity Analysis for the Indicators of Stochastic Type}

The purpose of getting the indicator's sensitivity includes: 1) acquire the weightiness of indicator and determine

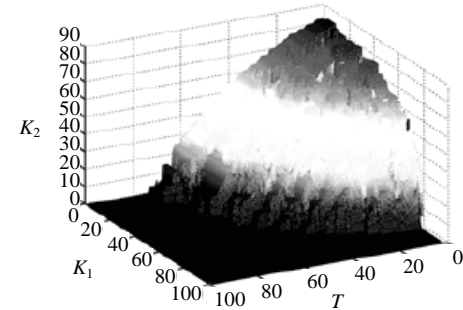

(a)

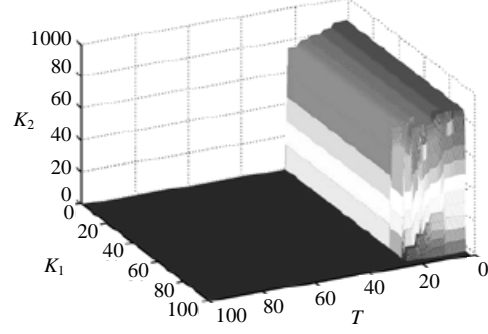

(b)

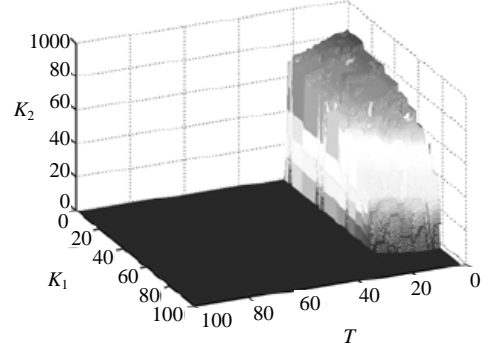

(c)

Figure 2. (a) MIRL1 for the stealth aircraft of type A; (b) MIRL2 for the type B; (c) the intersection of MIRL1 and MIRL2 acquired by the approaching method. 
which indicator is critical; 2) more carefully deal with the uncertain and sensitive context or system capability indicators; and 3) optimize system.

The sensitivity analysis is realized by the perturbation analysis. This paper uses the analytic method to acquire the indicator's sensitivity. In addition, every indicator is normalized within a common range for simplicity.

Definition 1. Denote the sensitivity of indicator $p_{i}$ as $S_{i}$ and its definition is

$$
S_{i}=\frac{\partial E}{\partial e_{i}}=\frac{\partial\left(\int_{P l} f(p, e) \mathrm{d} p\right)}{\partial e_{i}} \approx \frac{\partial\left(\sum_{j=1}^{N} \int_{H_{j}} f(p, e) \mathrm{d} p\right)}{\partial e_{i}},
$$

where $p l \approx \bigcup_{j=1}^{N} H_{j}$ and $e_{i}$ is the expected value of the system capability indicator $p_{i}$ (stochastic variable) and also the perturbation variable.

Definition 2. Denote the relativity between indicator $p_{i}$ and $p_{j}$ as $R_{i j}$.

$$
\begin{gathered}
\text { If } i=j, R_{i j}=\frac{\partial^{2} E}{\partial e_{i}^{2}} ; \\
\text { If } i \neq j, R_{i j}=\frac{\partial^{2} E}{\partial e_{i} \partial e_{j}} ;
\end{gathered}
$$

Definition 3. Possibility measure [13]. $\theta$ is assumed as a non-empty set and $P(\theta)$ as the power set of $\theta$. If Pos meets three axioms, i.e., $\operatorname{Pos}(\theta)=1, \operatorname{Pos}(\Phi)=0$, and $\operatorname{Pos}\left\{\cup_{i} A_{i}\right\}=\sup _{i} \operatorname{Pos}\left\{A_{i}\right\}\left(A_{i} \in P(\theta)\right)$, it is called possibility measure.

Definition 4. System effectiveness based on the fuzzy indicators and possibility measure $E_{f p}$. The expression of $E_{f p}$ is $E_{f p}=\operatorname{Pos}(p \in P l)$, where $p$ is multi-dimensional fuzzy indicator and $P l$ is the requirement locus. The value of $E_{f p}$ can be acquired by fuzzy simulation method which is detailed as follows.

Step 1 Set $L=a$ and $a$ is a very small estimate of $L$.

Step 2 According to subordinating fuction of $p_{i}$, uniformly produce $u_{i}(i=1,2, \cdots, n)$ from $a$-cut set and let $u=\left(u_{1}, u_{2}, \cdots, u_{n}\right)$.

Step 3 Set $u=u_{1}\left(p_{1}\right) \wedge u_{2}\left(p_{2}\right) \wedge \cdots \wedge u_{n}\left(p_{n}\right)$.

Step 4 If $p \in P l$ and $L<u$, set $L=u$.

Step 5 Repeat step 2 to $4 N$ times.

Step 6 If $E_{f p}=L$, return $E_{f p}$.

Credibility, which reveals the possibility and impossibility simultaneously, is a novel mode describing fuzzy relation. So, as a measure, credibility is more reasonable than possibility. The definition of credibility measure is stated as follows.

Definition 5. Credibility measure [13]. With the assumption that $(\theta, P(\theta), P o s)$ is a possibility space and $A$ is the element of power set $P(\theta)$, credibility measure $\operatorname{Cr}\{A\}$ is defined as

$$
\operatorname{Cr}\{A\}=\frac{1}{2}\left(\operatorname{Pos}\{A\}+\left(1-\operatorname{Pos}\left\{A^{c}\right\}\right)\right) .
$$

Definition 6. System effectiveness based on the fuzzy indicators and credibility measure $E_{f c}$. The expression of $E_{f c}$ can be stated as $E_{f c}=C r(p \in P l)=\frac{1}{2}(\operatorname{Pos}\{p \in P l\}+(1-\operatorname{Pos}\{p \notin P l\}))$, where $p$ is multidimensional fuzzy indicator and $P l$ is the requirement locus. Similarly, it can be acquired by fuzzy simulation method.

Step 1 Uniformly produce $p^{(k)}$ from $p$ and let $v_{k}=\operatorname{Pos}\left\{p^{(k)}\right\}(k=1,2, \cdots, N)$ if $\operatorname{Pos}\left\{p^{(k)}\right\} \geq \varepsilon$, where $\varepsilon$ is a very small number. 
Step 2 Let

$$
E_{f c}=\frac{1}{2}\left(\max _{1 \leq k \leq N}\left\{v_{k} \mid p^{(k)} \in P l\right\}+\min _{1 \leq k \leq N}\left\{1-v_{k} \mid p^{(k)} \notin P l\right\}\right)
$$

and then return $E_{f c}$.

For the above two algorithms, it is important to judge whether the $p$ is within $P l$ or not. And based on the tree structure output of partitioning algorithms introduced in the first one of this series papers, it can be quickly judged whether the $p$ is within $\mathrm{Pl}$ or not. The judging procedure is detailed as follows. Firstly, judge whether the $p$ is within the top MEH (root of the tree) or not. If it is not, it is not within $P l$ either and the judging procedure ends. If $p$ is within the MEH, judge whether $p$ is within the next-level MIH or not. If $p$ is within the MIH, it is also within $\mathrm{Pl}$ and the judging procedure ends. If it is not, judge whether $p$ is within one certain MEH of this level or not. If $p$ is contained by one $\mathrm{MEH}$, continue the recursive searching procedure from this MEH. If searching reaches the bottom of the tree and no one MIH containing $p$ is found, it means that this $p$ is not within $\mathrm{Pl}$.

\subsection{Effectiveness Analysis Based on Fuzzy MIRL}

Definition 7. Fuzzy monotonic indicator requirement locus (FMIRL) $f p l(\lambda) . \tilde{R}$ is a fuzzy set on a certain domain; $\tilde{R}_{\lambda}$ is the l-cut set of $\tilde{R}$; and $f p l(\lambda)$ is the effective range in $P$ (MIS) which meets the requirement $\tilde{R}_{\lambda}$. These concepts are now illustrated by an example. For an air defense system, the mission requirement measure (fuzzy set $\tilde{R}$ ) is killing the enemy aircraft. The domain is the kill probability. Set a $l$ to determine the $\tilde{R}_{\lambda} \cdot \tilde{R}_{\lambda}$ is a requirement measure of kill probability that must be larger than a certain value.

The fuzzy system capability indicator (assumed to be stochastic) effectiveness analysis model is introduced as follows. $f p l(\lambda)$ can be determined according to $l$. If $\lambda_{1} \leq \lambda_{2}$, we can get the corresponding conclusion, i.e., $\tilde{R}_{\lambda_{2}} \subseteq \tilde{R}_{\lambda_{1}}$ and $f p l\left(\lambda_{2}\right) \subseteq f p l\left(\lambda_{1}\right)$ according to the monotonic assumption.

$$
\begin{gathered}
E_{f} \text { (Fuzzy) } \int_{p l} f(p) \mathrm{d} p=\underset{0 \leq \lambda \leq 1}{\vee}\left(\lambda \wedge \int_{f p l(\lambda)} f(p) \mathrm{d} p\right) \text { and } f p l\left(\lambda_{1}\right) \supseteq f p l\left(\lambda_{2}\right) ; \\
\int_{f p l\left(\lambda_{1}\right)} f(p) \mathrm{d} p \geq \int_{f p l\left(\lambda_{2}\right)} f(p) \mathrm{d} p \text { when } \lambda_{1} \leq \lambda_{2} \text {. So, } E_{f}=\lambda^{*}=\int_{f p l\left(\lambda^{*}\right)} f(p) \mathrm{d} p \text {. And } E_{f} \text { can be solved rapidly by }
\end{gathered}
$$

the binary method. The fuzzy effectiveness analysis model is not only suitable for the fuzzy requirement but also able to decrease misclassification rate of the boundary points especially when the system capability indicator values are distributed near the boundary of MIRL.

\section{MIS-Based System Capability Indicator Optimization Design}

\subsection{Budget-Oriented Optimization Design}

Budget-oriented capability indicator optimization design will be discussed in this section. In order to make a system meet a certain requirement, there are usually many development "paths" with different cost for system capability indicators. Figure 3 gives three linking paths (i.e., OA, OB and OC) between point $\mathrm{O}$ representing the initial system capability and the requirement locus. And point $O$ is assumed to be out of the requirement locus. Certainly, besides these three paths, there exist countless other linking paths between point $\mathrm{O}$ and requirement locus. The purpose of capability indicator optimization design is to find out the path with the minimal budget or least time cost. In this paper, the optimization model is used to get the optimized expectation values of the statistical capability indicators according to practical problems. For simplicity, all the capability indicators are assumed to be mutually independent and have fixed variances (i.e., every capability indicator can be developed independently). And the optimization design model is:

$$
\begin{aligned}
& \operatorname{Min}\left(C\left(P_{0}, P_{e}\right)\right) \\
& \text { s.t }: E \geq K, E=\int_{P l} f\left(p, P_{e}\right) \mathrm{d} p .
\end{aligned}
$$

In this model: $f\left(p, P_{e}\right)$ is union probability density function and $P_{e}$, as the optimization variable, repre- 


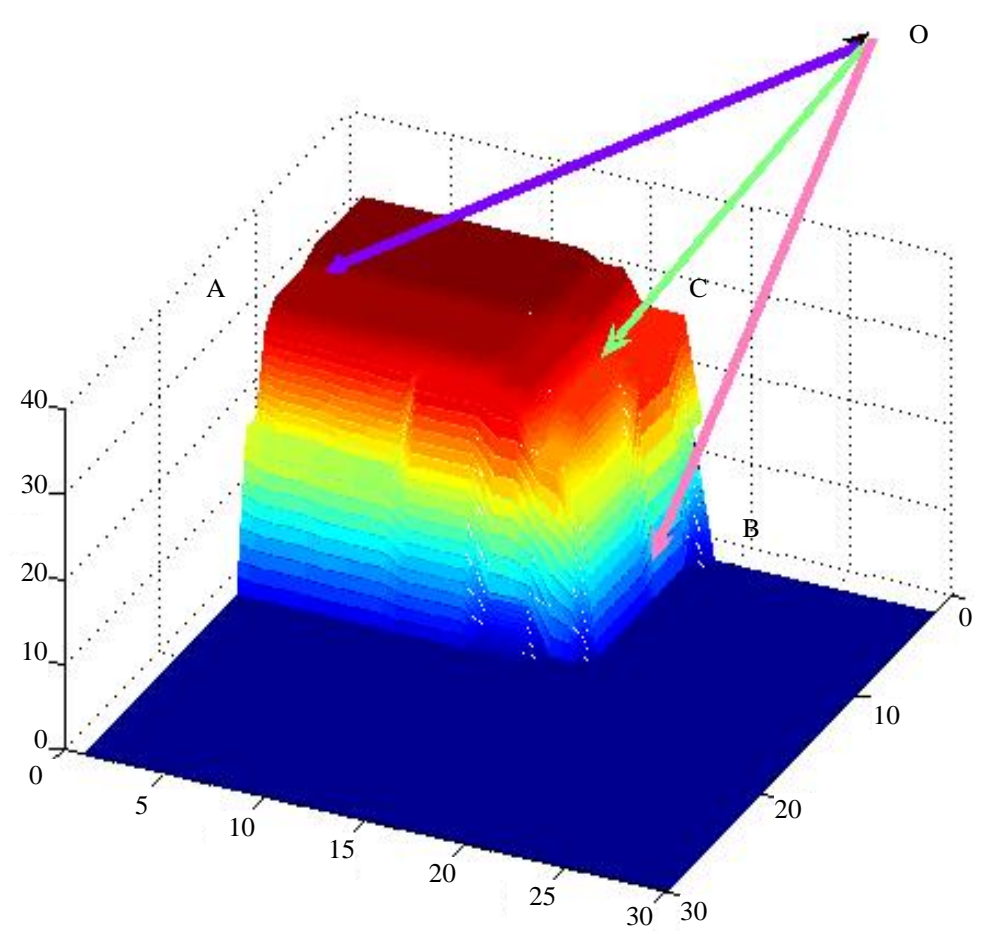

Figure 3. The paths to monotonic indicators requirement locus.

sents the expectation value of $f\left(p, P_{e}\right) ; p l, E$, and $K$ respectively represent the MIRL (monotonic indicators requirement locus), the effectiveness value, and the capability indicator effectiveness requirement threshold; $C\left(P_{0}, P_{e}\right)$ represents the budget-cost function of capability indicator varying from $P_{0}$ to $P_{e}$. The $P_{e}$ which meets the effectiveness requirement and has the least budget can be found through optimization. The corresponding duality programming is

$$
\begin{aligned}
& \operatorname{Max}(E) \\
& \text { s.t }: C\left(P_{0}, P_{e}\right) \leq C_{K}, E=\int_{P l} f\left(p, P_{e}\right) \mathrm{d} p .
\end{aligned}
$$

This model will be used to get the maximized capability indicator effectiveness value with the budget being less than $C_{K}$.

\subsection{Time-Oriented Optimization Design}

Capability development is usually a time-consuming work. The following optimization model is able to find the path from the original capability point to MIRL with the least time.

$$
\begin{aligned}
& \operatorname{Min}\left(\operatorname{Max}\left(T_{i}\left(P_{0}, P_{e}\right)\right)\right), 1 \leq i \leq M \\
& \text { s.t }: E \geq K, E=\int_{P l} f\left(p, P_{e}\right) \mathrm{d} p
\end{aligned}
$$

In this model: $T_{i}\left(P_{0}, P_{e}\right)$ represents time-cost function of the ith capability indicator varying from $P_{0}$ to $P_{e}$ and $M$ represents the total number of capability indicators. This model can also be transformed into the following programming model,

$$
\begin{aligned}
& \operatorname{Min}(\lambda) \\
& \text { s.t.: } T_{i}\left(P_{0}, P_{e}\right) \leq \lambda ., 1 \leq i \leq M \\
& \quad E \geq K, E=\int_{P l} f\left(p, P_{e}\right) \mathrm{d} p
\end{aligned}
$$


Similarly, the dual programming can be changed into

$$
\begin{aligned}
& \operatorname{Max}(E), E=\int_{P l} f\left(p, P_{e}\right) \mathrm{d} p \\
& \text { s.t: } T_{i}\left(P_{0}, P_{e}\right) \leq T_{K}, 1 \leq i \leq M
\end{aligned} .
$$

\section{A Notional Example: Number Optimization of Missiles Available}

In this section, some models and algorithms mentioned above are used to program missile available numbers of six types so as to meet the mission requirement with the least budget cost.

\subsection{Problem Context}

Ten types of opponent targets (i.e., T1, T2, T3, T4, T5, T6, T7, T8, T9, and T10) are assumed to be destroyed to win the air superiority. Target-attacking missile types are assumed to be type A, type B, type C, type D, type E, and type F.

Because missile production cost is usually very large, so the missile number available of each type is an important capability indicator. The capability indicator optimization design will be carried out in a MIS with six dimensions (i.e., six missile available number indicators). The required number of one certain type missile, when only this type of missile is used to destroy one target of a certain type, is presented in the following table. "No" in the table means that type of missile can not be assigned to attack the corresponding type of target.

If there exists solution meeting the following equality and inequalities group, it means that the missile number available of each type can satisfy the mission requirement.

$$
\left\{\begin{array}{l}
\sum_{i=1}^{6} \frac{x_{i j}}{R_{i j}}=1,1 \leq j \leq 10 \\
\sum_{j=1}^{10} x_{i j} \leq W_{i}, 1 \leq i \leq 6 \\
x_{i j} \geq 0
\end{array}\right.
$$

In the above equation and inequalities group, $x_{i j}, R_{i j}$, and $W_{i}$ respectively represent the number of the ith type of missile assigned to the $j$ th type of target, the required number of the ith type missile to destroy $j$ th type target, and the number available of ith type missile.

If the number available of each type of missiles all meet the mission, the requirement measure $u$ is set to 1 , otherwise, set to 0 . And the function $u=f\left(W_{1}, \cdots, W_{6}\right)$ is obviously monotonic.

\subsection{Capability Indicator Analysis and Optimization Design}

The MIS-based capability indicator analysis and optimization design is performed according to the following steps.

1) Construct the MIS, i.e., select all the MIS dimensions and standardize every dimension value range into the range $[0,100]$.

2) Create the monotonic function representing the relation between the capability indicators and requirement measure. Actually, the function $u=f\left(W_{1}, \cdots, W_{6}\right)$ is already put forward in section 4.1.

3) Apply the partitioning algorithm to acquire the MIRL, i.e., accomplish capability indicators requirement analysis.

4) Use the capability indicators effectiveness model (introduced in section 2.2) to judge whether the present capability meet the requirement or not. If not, go to step5 to perform optimization design, else stop capability indicator analysis and optimization design.

5) Trade off missile number for each type with the optimization model introduced in section 3.1.

Based on the data of Table 1, the MIRL shown in Figure 4 is acquired by the partitioning algorithm. The numbers of six types of missiles (i.e., type A, B, C, D, E, and F) available is respectively assumed to be 14 - 26 number unit, 14 - 26 number unit, 12 - 29 number unit, 14 - 26 number unit, 14 - 26 number unit, and 12 - 29 
number unit.

All the numbers of six types of missiles available are assumed to be subject to normal distribution and according to $6 \sigma$ principle, the corresponding distribution functions can be approximately stated as follows:

$$
\begin{aligned}
& f_{A}\left(N_{A}\right)=\frac{1}{\sqrt{(2 \pi)} 2} \mathrm{e}^{\frac{-\left(N_{A}-20\right)^{2}}{8}}, f_{B}\left(N_{B}\right)=\frac{1}{\sqrt{(2 \pi)} 2} \mathrm{e}^{\frac{-\left(N_{B}-20\right)^{2}}{8}}, f_{C}\left(N_{C}\right)=\frac{1}{\sqrt{(2 \pi)} 3} \mathrm{e}^{\frac{-\left(N_{C}-20\right)^{2}}{18}} \\
& f_{D}\left(N_{D}\right)=\frac{1}{\sqrt{(2 \pi)} 2} \mathrm{e}^{\frac{-\left(N_{D}-20\right)^{2}}{8}}, f_{E}\left(N_{E}\right)=\frac{1}{\sqrt{(2 \pi)} 2} \mathrm{e}^{\frac{-\left(N_{E}-20\right)^{2}}{8}}, f_{F}\left(N_{F}\right)=\frac{1}{\sqrt{(2 \pi)} 3} \mathrm{e}^{\frac{-\left(N_{F}-20\right)^{2}}{18}}
\end{aligned}
$$

Furthermore, the budget costs of six types of incremental missiles are assumed to be evaluated with the following functions:

$$
\begin{aligned}
& C(N a)=\left\{\begin{array}{ll}
25 N a & N a \geq 40 \\
15 N a & N a<40
\end{array} ; \quad C(N b)=\left\{\begin{array}{ll}
15 N b & N b \geq 10 \\
10 N b & N b<10
\end{array} ; \quad C(N c)=\left\{\begin{array}{ll}
25 N c & N c \geq 30 \\
15 N c & N c<30
\end{array} ;\right.\right.\right. \\
& C(N d)=\left\{\begin{array}{ll}
25 N d & N d \geq 30 \\
15 N d & N d<30
\end{array} ; C(N e)=\left\{\begin{array}{ll}
20 N e & N e \geq 20 \\
10 N e & N e<20
\end{array} ; C(N f)=\left\{\begin{array}{ll}
25 N f & N f \geq 25 \\
15 N f & N f<25
\end{array} .\right.\right.\right.
\end{aligned}
$$

In these functions: $\mathrm{Na}, \mathrm{Nb}, \mathrm{Nc}, \mathrm{Nd}, \mathrm{Ne}$, and $\mathrm{Nf}$ respectively represent the incremental missile numbers of the type A, B, C, D, E, and F; $C(N a), C(N b), C(N c), C(N d), C(N e)$, and $C(N f)$ respectively represent the budget costs of the incremental missiles of type A, B, C, D, E, and F.

If the effectiveness value $E$ (introduced in section 2.2) is required to be larger than 0.9 , the optimized in-

\begin{tabular}{|c|c|c|c|c|c|c|c|c|c|c|}
\hline target missile & $\mathrm{T} 1$ & $\mathrm{~T} 2$ & T3 & $\mathrm{T} 4$ & $\mathrm{~T} 5$ & T6 & $\mathrm{T} 7$ & T8 & T9 & $\mathrm{T} 10$ \\
\hline Type A & 20 & 45 & 12 & 18 & 30 & 6 & No & No & No & No \\
\hline Type B & 18 & 75 & 18 & 14 & 30 & 8 & No & No & No & No \\
\hline Type C & 15 & 60 & 12 & No & 20 & No & No & No & No & No \\
\hline Type D & No & No & No & No & No & No & 75 & 45 & 24 & 60 \\
\hline Type E & No & No & No & No & No & No & 45 & 37 & 24 & 40 \\
\hline Type F & No & No & No & No & No & No & No & 45 & 20 & 40 \\
\hline
\end{tabular}
cremental numbers of six types of missiles can be acquired through conducting the following programming model.

Table 1. The required number of a certain type missile to destroy a certain type target.

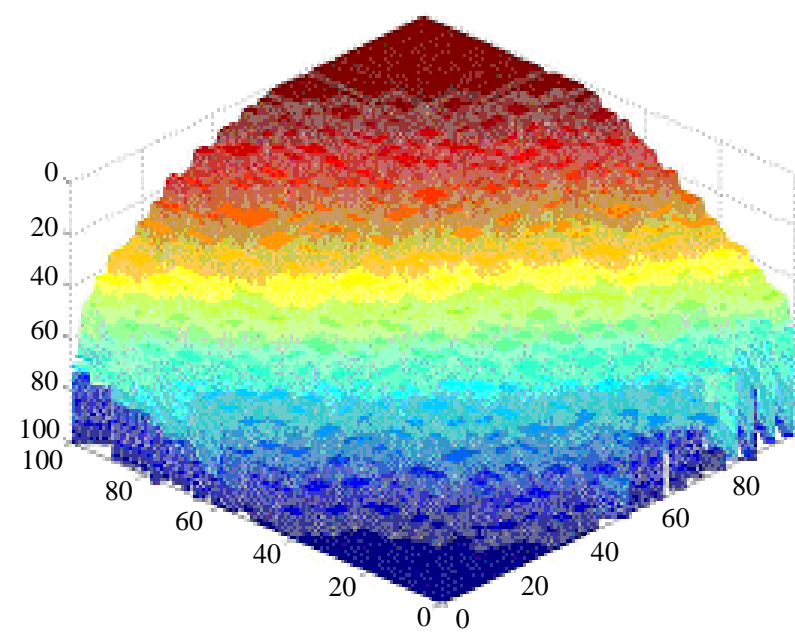

(a)

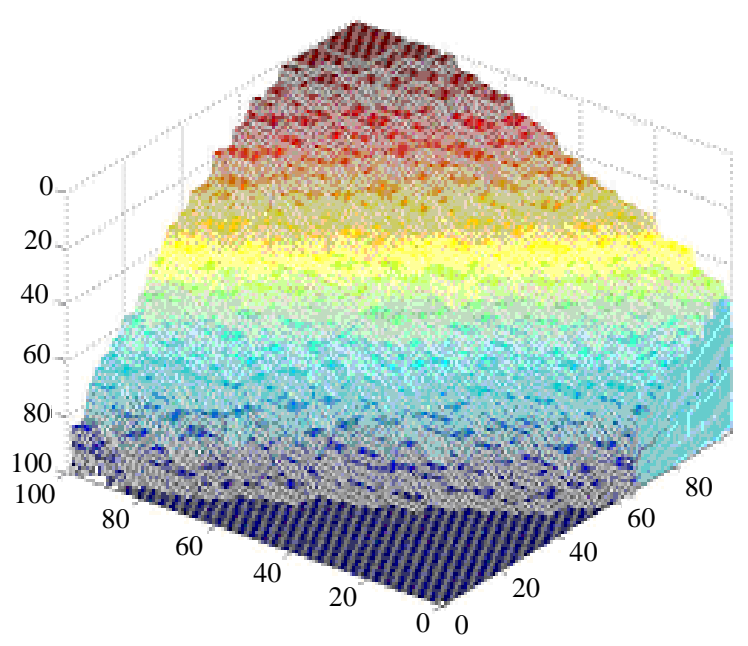

(b)

Figure 4. (a) The MIRL of missile type A, B, and C; (b) The MIRL of missile type D, E, and F. 


$$
\begin{aligned}
& \operatorname{Min}(C(N a)+C(N b)+C(N c)+C(N d)+C(N e)+C(N f)) \\
& \text { s.t. } E=\int_{P L} f_{A}(N a+20,2) f_{B}(N b+20,2) f_{C}(N c+20,3) f_{D}(N d+20,2) f_{E}(N e+20,2) f_{F}(N f+20,3) \mathrm{d} N_{A} \cdots \mathrm{d} N_{F} \\
& \quad \geq 0.9
\end{aligned}
$$

In this model:

$$
f_{A}(N a+20,2), f_{B}(N b+20,2), f_{C}(N c+20,3), f_{D}(N d+20,2), f_{E}(N e+20,2) \text {, and } f_{F}(N f+20,3)
$$

respectively represent the probability density functions of missile numbers of type A, B, C, D, E, and F; $N a+20, N b+20, N c+20, N d+20, N e+20$, and $N f+20$ respectively represent the expectation values of six distribution functions; $N a, N b, N c, N d, N e$, and $N f$ respectively represent the incremental missile numbers of type A, B, C, D, E, and F. The initial numbers of six types of missiles are all 20 and variances of six distribution functions are 2 or 3.

Through solving the programming model mentioned above, we get such conclusions that the optimized incremental missile numbers of type A, B, C, D, E, and F should be 28 number unit, 28.2 number unit, 27.9 number unit, 25.7 number unit, 36.5 number unit, and 35.9 number unit and the optimized least budget cost will be 3275 (cost unit).

\section{Conclusion and Perspective}

This series of papers propose and introduce a novel model, i.e., MVS (monotonic vector space) model, in which there exist some monotonic mappings. Many MVS operations (such as partitioning operation, synthesizing operation, sampling operation, etc.) can be directly used to solve related practical problems, which have been elaborated in the first one of this series papers. Based on MVS, some system capability indicator analysis and optimization models (e.g., requirement analysis model and effectiveness analysis model) are put forward and used to perform capability indicator analysis and optimization design in the second one of this series of papers. The future work for MVS model includes: 1) improving the present operation algorithms and developing other types of operations (such as partitioning operation in non-deterministic discrete or non-deterministic continuous MVS), which is of great significance to practical problems; 2) extending the application of the MVS model in other fields such as imaging processing and multi-object programming, etc.

\section{Funding}

Supported by NSFC, China, PRC, Grant No 70871120.

\section{REFERENCES}

[1] WSEIAC Report, “AFSC TR-65-2, Vol. 1-3, Final Report of Task Group 2, Preciction Measurement,” 1965.

[2] C. L. Hwang, et al., “Multiple Attribute Decision Making,” Springger-Verlag, Berlin, 1981. http://dx.doi.org/10.1007/978-3-642-48318-9

[3] W. D. Cook and M. A. Kress, “A Multiple-Criteria Composite Index Model for Quantitative and Quantitative Data,” European Journal of Operation Research, Vol. 78, No. 3, 1994, pp. 367-379. http://dx.doi.org/10.1016/0377-2217(94)90046-9

[4] T. L. Saaty, “The Analytic Hierarchy Process,” McGraw-Hill, 1980.

[5] V. Bouthonnier and A. H. Levis, "System Effectiveness Analysis of C3 Systems," IEEE Transaction on Systems, Man, and Cybernetics, Vol. 14, No. 1, 1984, pp. 48-54. http://dx.doi.org/10.1109/TSMC.1984.6313268

[6] P. K. Davis, “Analytic Architecture for Capabilities-Based Planning, Mission-System Analysis, and Transformation,” RAND MR-1513-OSD, 2002.

[7] M. C. Bohner, “Computer Graphics for System Effectiveness Analysis,” M.S. Thesis, Massachusetts Institute of Technology, Dept. of Electrical Engineering and Computer Science, 1986.

[8] X. F. Wu, "SEA Methods and Its Application in C3I System(4)-System Effectiveness Analysis,” System Engineering Theory and Practice, Vol. 2, No. 2, 1999, pp. 44-49.

[9] B. A. Nixon, "Management of Performance Requirements for Information Systems," IEEE Trans on Software Engineering, Vol. 26, No. 12, 2000..

[10] P. H. Cothier and A. H. Levis, “Timeliness and Measures of Effectiveness in Command and Control,” In: S. J. Andriole and S. 
M. Halpin, Eds., Information Technology for Command and Control, IEEE Press, New York, 1991.

[11] N. M. Paul, “Evolution Equations of C3I: Toward a New Understanding of Military of Combat,” In: Application of Artificial Intelligence to Command \& Control Systems IEE Computing Series 13, Peter Peregrinus Ltd..

[12] L.Washington, “Effectiveness Analysis of Flexible Manufacturing Systems LIDS-TH-1430,” 1985.

[13] B. Liu, “Uncertain Programming with Applications,” Tsinghua Springer Press, Beijing, 2003. 